\title{
Strategies for Decoupling Internal and External Dynamics Resulting From Inter-Arm Passive Component Tolerances in HVDC-MMC
}

\author{
Shuren Wang \\ Dept. of Electronic and Electrical \\ Engineering \\ University of Strathclyde \\ Glasgow, UK \\ shuren.wang@strath.ac.uk \\ Derrick Holliday \\ Dept. of Electronic and Electrical \\ Engineering \\ University of Strathclyde \\ Glasgow, UK \\ derrick.holliday@strath.ac.uk
}

\author{
Grain Adam \\ Dept. of Electronic and Electrical \\ Engineering \\ University of Strathclyde \\ Glasgow, UK \\ grain.adam@strath.ac.uk \\ Barry Williams \\ Dept. of Electronic and Electrical \\ Engineering \\ University of Strathclyde \\ Glasgow, UK \\ barry.williams@strath.ac.uk
}

\author{
Ahmed Massoud \\ Dept. of Electrical Engineering \\ Qatar University \\ Doha, Qatar \\ ahmed.massoud@qu.edu.qa
}

\begin{abstract}
Modular Multilevel Converter (MMC) performance may be adversely affected by passive component tolerances, such as submodule capacitance and arm inductance variations. Depending on control strategies, the differences in equivalent capacitances and/or inductances of the upper and lower arms of one phase-leg can cause unequal power distribution between upper and lower arms. Assuming passive component tolerances ranging between $\pm 10 \%$, this paper presents a comprehensive assessment of the internal/external coupling effects due to the passive component tolerances within one phase-leg, under the control of common MMC balancing methods. A novel control strategy is proposed to suppress the fundamental component that arises in the dc-link current due to such tolerances, and its effectiveness is demonstrated via simulation and experimentation. The investigation shows that voltage-based common and differential mode balancing control provides effective ac offset suppression while the proposed method offers superior performance in terms of dc-link fundamental current ripple suppression.
\end{abstract}

Keywords-Modular Multilevel Converter (MMC), internal control, voltage and power balance, component tolerances

\section{INTRODUCTION}

As a preferable solution for point-to-point and multiterminal HVDC transmission, the Modular Multilevel Converter (MMC) has attracted considerable attention due to its salient features of modularity, scalability, low semiconductor losses, and reduced distortion of ac side waveforms [1].

The MMC control system is more complex than that of the conventional voltage source converter. The use of floating distributed capacitors in multilevel converters results in complex internal dynamics, which necessitates the adoption of a complex multi-layer control system. MMC commonmode current, which consists of dc and harmonics, acts as a link between the powers of the dc side and submodule (SM) capacitors. Also, the inner SM capacitor voltages affect the ac side voltage synthesis in terms of both differential and common mode ac voltage. To reduce such internal and external coupling, many control methods have been developed to suppress the circulating current and regulate capacitor voltages, independent of the dc-link voltage [2]. SM, arm and leg are three internal control layers within an MMC from the topology and function points of view. SM capacitor voltage balancing is essential for both semiconductor safety and arm and leg level performance. To manage the voltage differences between SMs within each arm, SM voltage balancing algorithms based on either centralized or individual measures are employed [3], [4]. Consequently, a decoupled internalexternal MMC characteristic is desirable as it improves output performance [5], [6]. Research effort has been invested into higher-level controllers (arm and leg level capacitor sum characteristics) [7], [8]. Usually, identical passive components are assumed. Although a large number of SMs may reduce the adverse effects of capacitance tolerance, component tolerance issues still exist; therefore, parametric uncertainty due to passive component tolerances should be taken into account during MMC design and maintenance [9]-[12]. The adverse effects on ac output voltage caused by MMC asymmetrical capacitance with three-level flying capacitor SMs are analyzed when an energy-based balancing approach is adopted [10]. The existence of fundamental frequency ripple in the dc-link, due to asymmetrical arm inductances is highlighted and a voltage-based active control method is suggested in [11]. However, no comprehensive analysis of the internal and external coupling effects due to capacitance and inductance tolerances has been investigated.

This paper considers the adverse effects of passive component tolerances on both the dc and ac sides, with voltage and energy-based balancing controllers. Moreover, a differential-mode voltage balancing control method reduces the fundamental component in the common-mode and dc-link currents of the MMC with significant passive component tolerances. However, its effectiveness is limited since it is not direct control and its control objective is to nullify the differential-mode capacitor voltage sums. Considering the random nature of the passive component tolerance distribution within one phase-leg of an MMC-HVDC system, this paper proposes a proportional-resonant (PR) based controller that operates at the fundamental frequency to suppress any fundamental circulating component that may arise in the common-mode currents. The effectiveness of the proposed method compared with existing control methods is presented. This paper is subsequently organized as follows. Section II provides a brief review of MMC fundamentals and then introduces the issues that arise due to passive component 


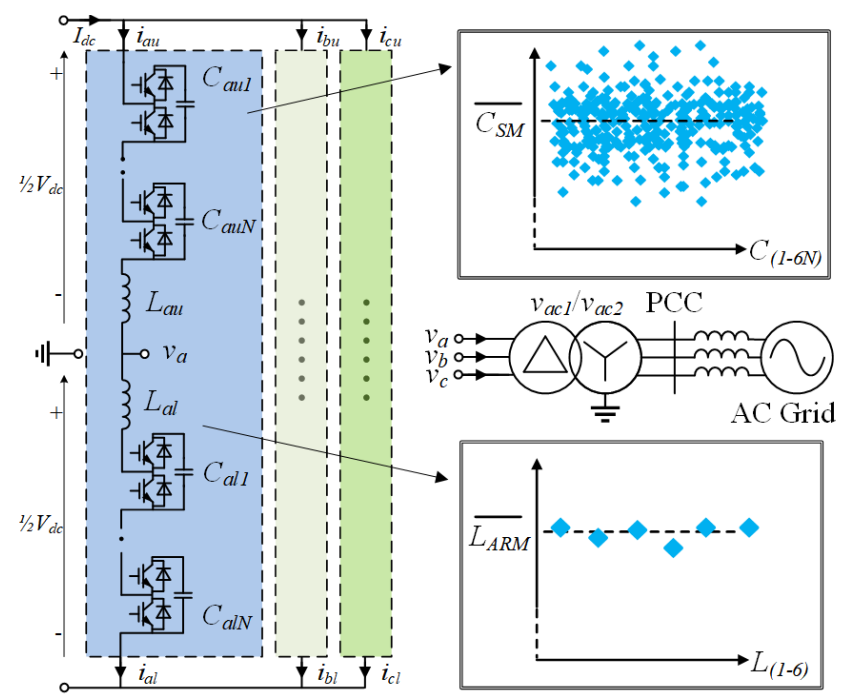

Fig. 1. MMC Topology configuration.

tolerances. Section III introduces inter-arm balancing methods and proposes a direct method of suppressing fundamental circulating current. Section IV uses simulations to assess the performances of control methods, and experimental results to validate the proposed direct control method compared to the indirect control method. Experimentation is performed on a grid-connected single-phase MMC prototype. Finally, section $\mathrm{V}$ summarizes the major findings of this paper.

\section{BASIC OPERATION AND PASSIVE COMPONENT TOLERANCES OF MMC}

Fig. 1 shows a three-phase half-bridge MMC, with $V_{d c}$ and $I_{d c}$ representing the dc bus voltage and current respectively. Each phase-leg includes upper and lower arms, and each arm consists of a reactor with an expected inductance $\overline{L_{A R M}}$ and $N$ series-connected SMs. Each SM consists of a capacitor with an expected capacitance $\overline{C_{S M}}$ and an IGBT-based half-bridge. The term circulating current represents the ac component of the common-mode current, $i_{c m}$, and is mainly caused by crossmodulation of the upper and lower arms, that is, the interaction of voltages, currents and switching functions. Strategies of the inner-arm SM voltage balancing and the second-order circulating current suppression have been widely discussed [2]-[5]. Generally, the MMC is tied to the ac grid with an interfacing transformer, as shown in Fig. 1.

Neglecting circuit parameter tolerances is a common practice to facilitate modeling, analysis and controller design of such a complex energy conversion system like the MMC. But, it is essential to take countermeasures to neutralize any potential implications of circuit parameter tolerances on MMC performance [10]. The reasons are as follows:

- With the large number of passive components involved, tolerances are inevitable and vary with many factors, as illustrated in Fig. 1. Also, the lifetimes should be taken into account;

- Although SM capacitor voltage balancing distributes the total dc voltage across each arm equally among the SMs, with switching devices of each SM only experiencing the SM capacitor voltage, asymmetric parameters may cause unequal power (or voltage) distribution between the upper and lower arms; thus, in

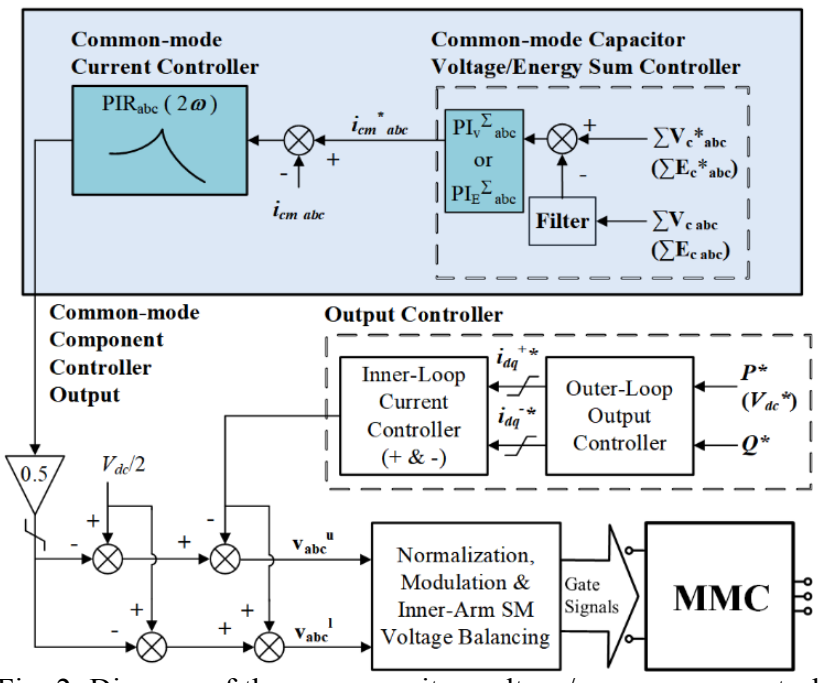

Fig. 2. Diagram of the arm capacitor voltage/energy sum control strategy without vertical balancing controller.

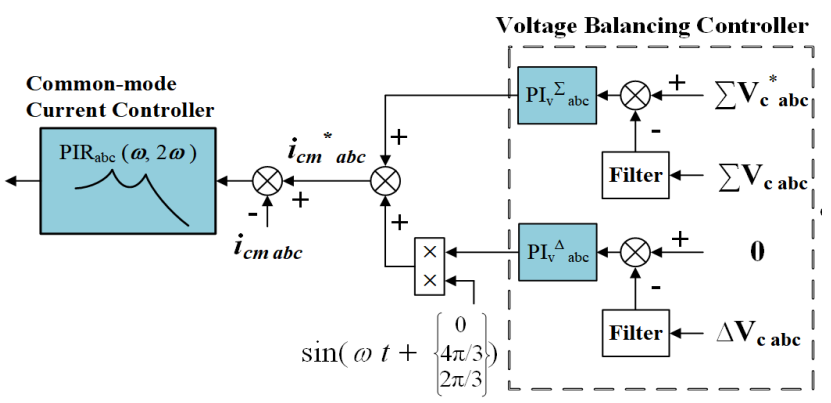

Fig. 3. Diagram of the differential-mode capacitor voltage sum controller.

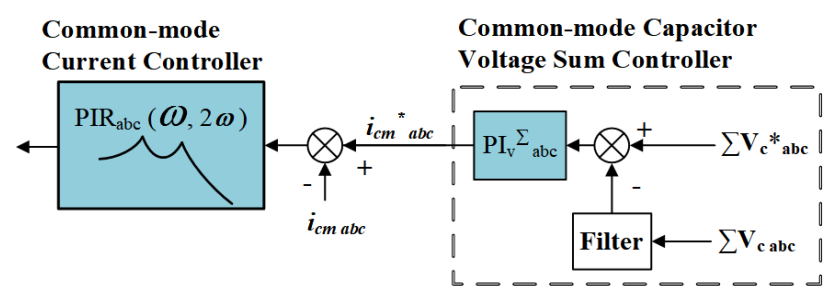

Fig. 4. Diagram of the proposed direct fundamental frequency circulating current suppressing method.

the current and voltage stresses of the switching devices and heat distribution; and

- Capacitance and inductance differences between arms tend to actuate unbalanced fundamental frequency common-mode currents, which can leak into the dclink and appear as undesirable current ripple.

This paper focuses on the tolerances between upper and lower arms within one phase-leg. Such vertical asymmetry has several operational implications, specifically, contamination of common-mode current by circulating current at fundamental frequency which increases semiconductor losses and generates fundamental frequency ripple in the dc side; and reduced exploitable modulation index range which generates a common-mode voltage in the ac output. In this paper, the sum and difference of arm capacitor voltage (energy) sums are referred to as the common and differential mode capacitor voltage (energy) sums of each phase-leg respectively. 
TABLE I. SIMULATION PARAMETERS

\begin{tabular}{|l|c|c|}
\hline \multicolumn{2}{|c|}{ System parameters } & Value \\
\hline DC voltage & $V_{d c}$ & $100 \mathrm{kV}$ \\
Rated power & $S$ & $100 \mathrm{MVA}$ \\
AC grid line to line voltage & $v_{a c 2}$ & $66 \mathrm{kV}$ \\
AC grid frequency & $F$ & $50 \mathrm{~Hz}$ \\
Transformer ratio & $v_{a c 1} / v_{a c 2}$ & $50 \mathrm{kV} / 66 \mathrm{kV}$ \\
Transformer leakage-inductance & $L_{T}$ & $0.2 \mathrm{pu}$ \\
Numbers of SMs per arm & $N$ & 50 \\
Expected arm inductance value & $L$ & $0.18 \mathrm{pu}$ \\
Expected SM capacitance value & $C$ & $6.7 \mathrm{mF}$ \\
Cable resistance per km & $R_{\text {cable }}$ & $10 \mathrm{~m} \Omega / \mathrm{km}$ \\
Cable inductance per km & $L_{\text {cable }}$ & $1.4 \mathrm{mH} / \mathrm{km}$ \\
Cable capacitance per km & $C_{\text {cable }}$ & $0.1 \mu \mathrm{F} / \mathrm{km}$ \\
\hline
\end{tabular}

(a) Method-A

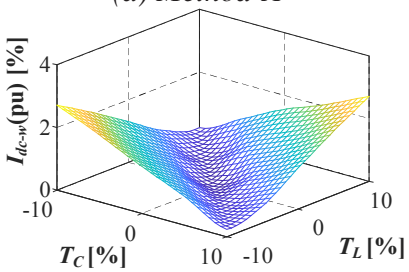

(c) Method-C

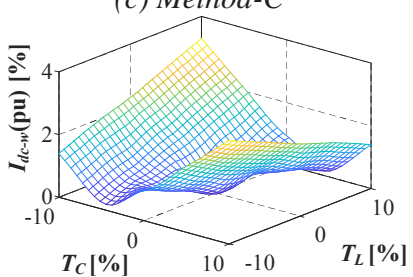

(b) Method-B

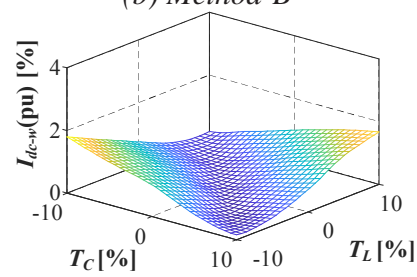

(d) Method-D

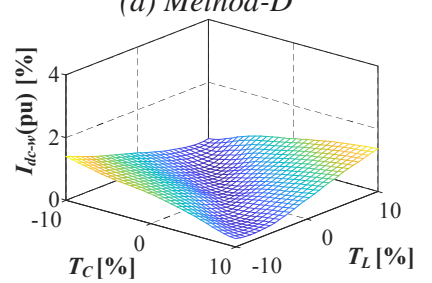

Fig. 5. Variation of fundamental frequency dc-link current ripple magnitude (pu) with SM capacitance tolerances $T_{C}$ and arm inductor tolerances $T_{L}$. (a) Method-A

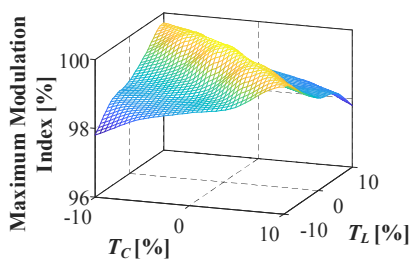

(c) Method-C

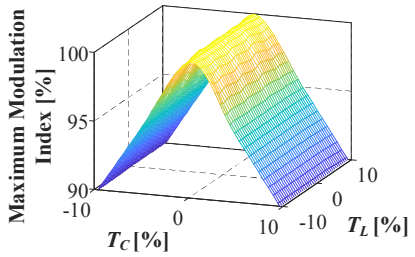

(b) Method-B

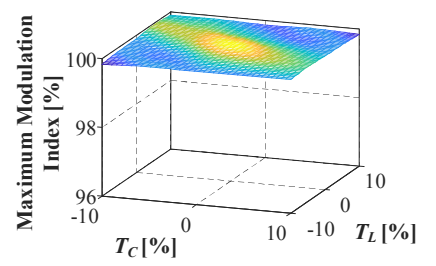

(d) Method-D

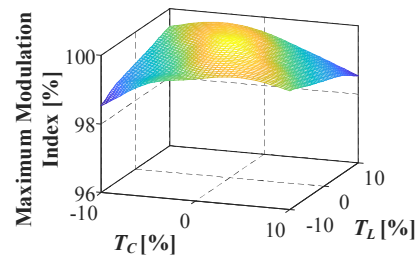

Fig. 6. Variation of maximum modulation index with SM capacitance tolerances $T_{C}$ and arm inductor tolerances $T_{L}$.

\section{MMC INTER-ARM BALANCING METHODS}

Fig. 2 shows the general structure of arm capacitor voltage (or energy) sum control without a vertical balancing part. The same ac output controller is inherited by the arm capacitor voltage sum control in Fig. 3 and Fig. 4, where a positive and negative sequence separation algorithm, outer-loop output controller and inner-loop current controller are enabled to generate 3-phase ac output voltage commands for the normalized modulation functions. In Fig. 2, regulation of average SM capacitor voltage (common-mode capacitor voltage sum) is achieved by the common-mode current controller plus the common-mode capacitor voltage/energy sum controller (where $\Sigma \mathrm{V}_{\mathrm{c}}$ and $\Sigma \mathrm{E}_{\mathrm{c}}$ represent the commonmode capacitor voltage and energy sums within one phase-leg respectively). To eliminate the steady-state dc mean value error and suppress the $2 \omega$ circulating current, a PIR (proportional integral and resonant) controller with a resonant frequency at $2 \omega$ is adopted [13]. Differential-mode capacitor voltage (or energy) sum control can be achieved by regulating arm active power using fundamental common-mode current injection together with another resonant frequency $\omega$ component and the generic control blocks are shown in Fig. 3.

Although each MMC arm outputs/absorbs zero net power during one period theoretically, tolerances of the passive components lead to different energy stored within the arm inductances or SM capacitors. Thus, the instantaneous power of the passive components deviates from nominal values at the fundamental frequency. As voltage (or energy) of each arm is usually controlled by corresponding inter-arm balancing controllers, such power imbalance will be mainly compensated by currents, in the form of fundamental frequency circulating currents. Thus, the vertical asymmetry between the upper and lower arms leads to odd order harmonics in the common-mode current, of which the actual amplitudes depend on a multiple of variables, such as cable length, control system bandwidth, etc. [14]. Without a dedicated controller, the inter-arm passive components can result in an adverse outcome, namely, the amplitude and phase angle of the fundamental common-mode currents of the three legs may be unbalanced, causing fundamental frequency oscillation in the dc-link and increased semiconductor losses. Additionally, depending on the control scheme, the vertical voltage difference may be deduced by the different energy distributions within one phase-leg, causing unbalanced vertical voltage when synthesizing the ac output voltage of MMC. All these violate the decoupled internal/external relationships.

The proposed direct suppressing method shown in Fig. 4 introduces and takes advantage of the fundamental frequency part of the PR-based common-mode current controller to detect and cancel the fundamental frequency components in the common-mode currents; thereby suppressing the dc-link current ripple. However, the potential disadvantage of the direct fundamental frequency circulating current suppressing method is that the maximum attainable modulation index is reduced due to the lack of vertical balancing control. Fortunately, the full modulation index range is not always used in practical operation.

\section{Controller Performance Evaluation}

Detailed parametric simulation studies are used to assess the performance of the internal and external decoupling control methods when passive component tolerances are considered. The findings of these studies are further validated by time-domain experimental waveforms.

\section{A. Simulation Results}

An MMC-based HVDC transmission station with a $40 \mathrm{~km}$ $\mathrm{dc}$ cable is simulated with the control methods in the previous section and parameters listed in Table I. The average tolerances of the SM capacitance $\left(C_{u}\right.$ and $\left.C_{l}\right)$ and arm inductance $\left(L_{u}\right.$ and $\left.L_{l}\right)$ of the upper and lower arms vary within $\pm 10 \%$ of the nominal. The MMC operating conditions remain the same, injecting $100 \mathrm{MW}$ into ac grid at unity power factor. 


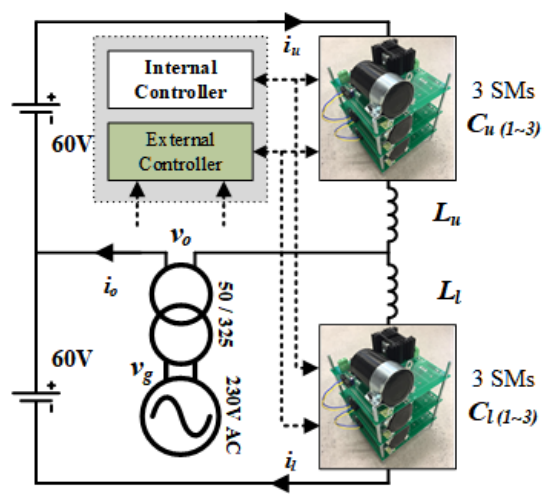

Parameters of Case I

$C_{u 1}=3.3 \mathrm{mF} ; C_{l l}=3.5 \mathrm{mF}$;

$C_{u 3}=3.7 \mathrm{mF} ; L_{u l}=2.8 \mathrm{mH}$.

$C_{l l}=3.6 \mathrm{mF} ; C_{l 3}=3.7 \mathrm{mF}$;

$C_{l 3}=3.6 \mathrm{mF} ; L_{l}=2.7 \mathrm{mH}$

Parameters of Case II

$C_{u 1}=3.6 \mathrm{mF} ; C_{1 L 2}=3.5 \mathrm{mF}$;

$C_{l l 3}=3.7 \mathrm{mF} ; L_{l l}=2.8 \mathrm{mH}$

$C_{l l}=3.6 \mathrm{mF} ; C_{l 2}=3.7 \mathrm{mF}$;

$C_{l 3}=3.6 \mathrm{mF} ; L_{l}=2.7 \mathrm{mH}$

Fig. 7. Experimental testing rig of MMC with three SMs per arm.

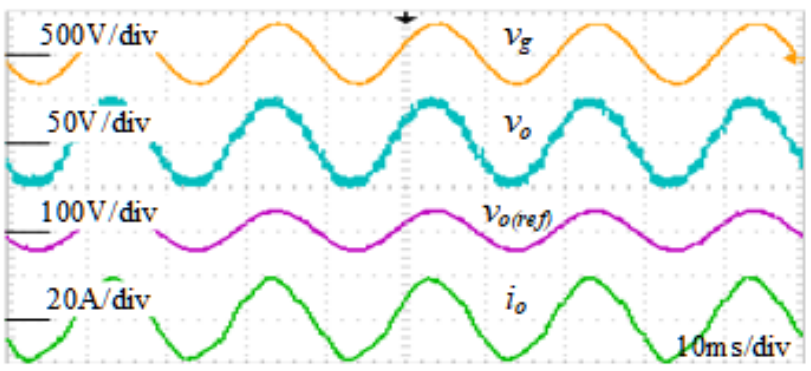

Fig. 8. Grid voltage, MMC ac output voltage and its reference, and MMC ac output current.

Four control methods considered in these parametric studies to regulate MMC internal dynamics are: Method-A: commonmode capacitor voltage sum control; Method-B: common and differential mode capacitor voltage sum balancing; Method$\mathrm{C}$ : common and differential mode capacitor energy sum balancing; and Method-D: common-mode capacitor voltage sum control with the proposed fundamental circulating current suppression.

Fig. 5 and Fig. 6 depict detailed quantitative comparisons of the fundamental frequency ripple in the dc current normalized by the mean value and attainable modulation range versus passive component tolerances, for the four methods. The main observations are:

- Fig. 5(a)-(d) show that certain combinations of passive component tolerances exhibit larger fundamental frequency ripple in the dc current. Fig. 5(b) and (c) reveal that Method-B exhibits better fundamental ripple suppression than Method-C;

- Fig. 5(d) displays the smallest fundamental frequency ripple among all methods;

- Fig. 6(a) and (d) show that with Methods A and D, the component tolerances reduce the linear modulation index range by $2 \%$ approximately, whilst Method-B retains maximum and nearly constant modulation index range for all tolerances, see Fig. 6(b). Fig. 6(c) shows the modulation index range of Method-C deteriorates rapidly and mainly with capacitance tolerance; and

- Fig. 6(d) shows that Method-D offers the best performance in term of fundamental frequency ripple suppression at the expense of a small reduction of the linear modulation range.

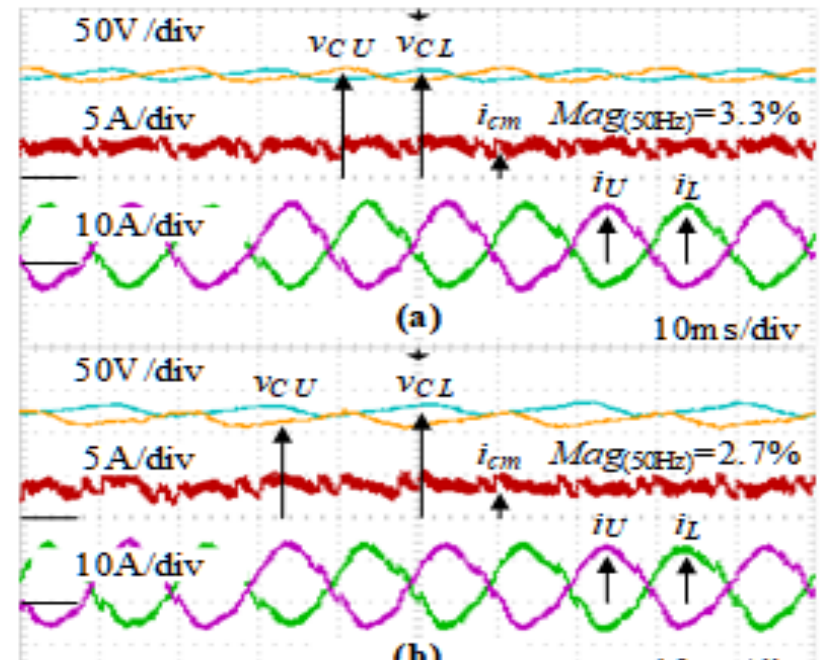

(b)

$10 \mathrm{~ms} / \mathrm{div}$

Fig. 9. Upper and lower capacitor voltage sum, common-mode current and upper and lower arm currents in case I: (a) Method-B; (b) Method-D.

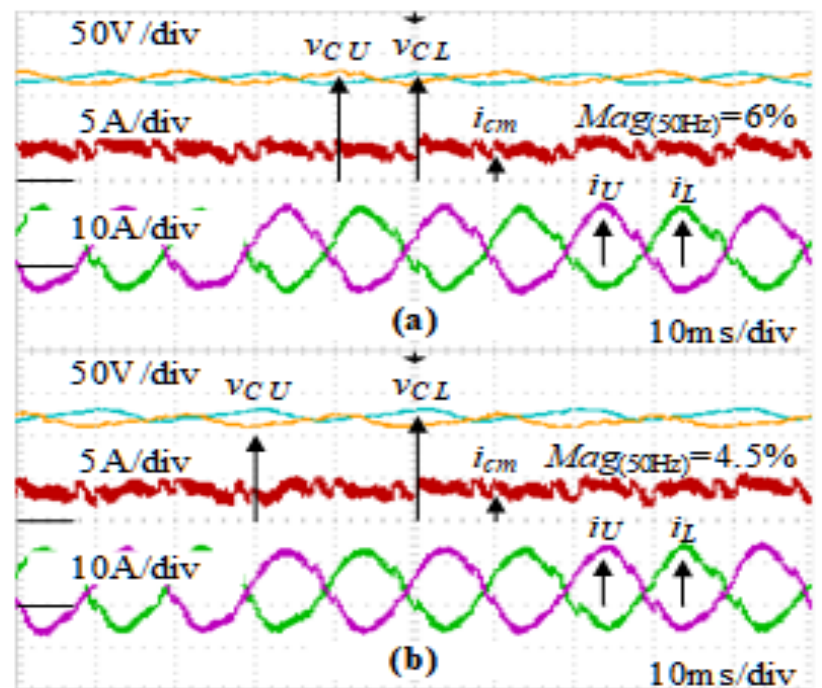

Fig. 10. Upper and lower capacitor voltage sum, common-mode current and upper and lower arm currents in case II: (a) Method-B; (b) Method-D.

\section{B. Experimental Validation}

A scaled-down single-phase prototype of grid-connected MMC with three SMs per arm and parameters in Fig. 7 is used to corroborate the findings of the parametric simulation studies, and the MMC injects $500 \mathrm{~W}$ at unity power factor into to ac grid. Internal control methods $\mathrm{B}$ and $\mathrm{D}$, which are the best performing control methods identified from the simulation studies, are validated. AC side waveforms are shown in Fig. 8, where grid voltage $v_{g}$, converter terminal voltage $v_{o}$, modulating signal $v_{o(\text { ref })}$ and output current $i_{o}$ indicate satisfactory operation with PWM-PD modulation at a $1 \mathrm{kHz}$ carrier frequency. The internal control scheme shown in Fig. 2 is derived based on theoretical relationships that describe the MMC common-mode quantities [15]. Moreover, with the scaled-down MMC experimental test rig parameters in Fig. 7, parameters of the internal-dynamics controllers implemented in the microprocessor are given in Table II. 
TABLE II. CONTROLLER PARAMETERS

\begin{tabular}{|c|c|c|}
\hline \multicolumn{2}{|l|}{ Items } & Value \\
\hline \multicolumn{2}{|l|}{ Common-mode current controller (PIR) } & \\
\hline Proptional gain & $K_{p}{ }^{c i r}$ & $1.2 \mathrm{pu} / \mathrm{A}$ \\
\hline Integral gain & $K_{i}^{c i r}$ & $0.8 \mathrm{pu} /(\mathrm{As})$ \\
\hline Resonant gain for the $1^{\text {st }}$-order component & $K_{i}{ }^{c i r 50}$ & $30 \mathrm{pu} /(\mathrm{As})$ \\
\hline Bandwidth for the $1^{\text {st }}$-order component & $\omega_{c}{ }^{c i r 50}$ & $0.05 \pi \mathrm{rad} / \mathrm{s}$ \\
\hline $1^{\text {st }}$-order resonant frequency & $\omega_{o}{ }^{c i r 50}$ & $100 \pi \mathrm{rad} / \mathrm{s}$ \\
\hline Resonant gain for the $2^{\text {nd }}$-order component & $K_{i}^{c i r 100}$ & $100 \mathrm{pu} /(\mathrm{As})$ \\
\hline Bandwidth for the $2^{\text {nd }}$-order component & $\omega_{c}{ }^{c i r l 100}$ & $0.2 \pi \mathrm{rad} / \mathrm{s}$ \\
\hline $2^{\text {nd }}$-order resonant frequency & $\omega_{o}{ }^{\operatorname{cir} 100}$ & $200 \pi \mathrm{rad} / \mathrm{s}$ \\
\hline \multicolumn{2}{|c|}{ Common-mode capacitor voltage sum controller } & \\
\hline Proptional gain & $K_{p}^{\text {VcSum }}$ & $0.1 \mathrm{~A} / \mathrm{V}$ \\
\hline Integral gain & $K_{i}^{V c S u m}$ & $10 \mathrm{~A} /(\mathrm{Vs})$ \\
\hline \multicolumn{2}{|c|}{$\begin{array}{l}\text { Differential-mode capacitor voltage sum controller } \\
\text { (for Method-B only) }\end{array}$} & \\
\hline Proptional gain & $K_{p}^{V c D i f}$ & $0.04 \mathrm{~A} / \mathrm{V}$ \\
\hline Integral gain & $K_{i}^{V c D i f}$ & $80 \mathrm{~A} /(\mathrm{Vs})$ \\
\hline
\end{tabular}

Fig. 9 and Fig. 10 show the upper and lower arm capacitor voltage sums, arm currents and their common-mode component for methods $\mathrm{B}$ and $\mathrm{D}$, under two passive parameter cases, I and II, respectively. The combination of capacitances and inductances in case II with substantial capacitance asymmetry results in larger fundamental frequency ripple of $6 \%$ and $4.5 \%$ for methods $\mathrm{B}$ and $\mathrm{D}$ respectively. Method-B suppresses fundamental current in the common-mode while maintaining strict upper and lower capacitor voltage sum balance. For this reason, Method-B manages to suppress fundamental frequency ripple to some extent and ensure even dc voltage distribution across upper and lower arms for a range of passive parameter tolerances, with no sacrifice of noticeable modulation index range or risk of dc voltage offset at the converter ac side. The FFT analysis of common-mode currents confirms that Method-D has better fundamental circulating current suppression performance than Method-B but at the expense of modest imbalance of upper and lower arm capacitor voltage sums. With Method-D, the worst-case voltage imbalance of mean dc value of $v_{C L}$ and $v_{C U}$ is about $9 \mathrm{~V}$ $( \pm 3.8 \%$ per SM). However, it is still sufficient for the ac output voltage synthesis under the operating condition at hand.

\section{CONCLUSION}

This paper analyzed the internal/external coupling effects caused by MMC inter-arm passive component tolerances under conventional and proposed balancing controllers. Investigation of the relationships between the fundamental dc current amplitude, modulation index linear range and passive component tolerances was presented. Detailed parametric simulation studies on a full-scale MMC that employs four different control methods and experimental results of scaleddown MMC prototype that employs the two control methods revealed the following:

- The performance of the energy-based method deteriorates faster with passive component tolerances, that is, the rapid growth of fundamental current ripple in dc link; arm voltage imbalance, and risking transformer saturation from dc voltage and current injection;

- The voltage-based method suppresses fundamental ripple in the dc-link to some extent, maintaining superior balanced arm voltage sums and maximum modulation index range; and
- The proposed method exhibits superior performance on dc-link current fundamental ripple suppression at the expense of a small reduction of linear modulation index range due to a small arm voltage sum imbalance.

\section{ACKNOWLEDGMENT}

This publication was made possible by NPRP grant NPRP (9-092-2-045) from the Qatar National Research Fund (a member of Qatar Foundation). The statements made herein are solely the responsibility of the authors.

\section{REFERENCES}

[1] M. A. Perez, S. Bernet, J. Rodriguez, S. Kouro, and R. Lizana, "Circuit topologies, modeling, control schemes, and applications of modular multilevel converters," IEEE Trans. Power Electron., vol. 30, no. 1, pp. 4-17, 2015.

[2] J. Wang, J. Liang, C. Wang, and X. Dong, "Circulating current suppression for MMC-HVDC under unbalanced grid conditions," IEEE Trans. Ind. Appl., vol. 53, no. 4, pp. 3250-3259, 2017.

[3] L. Xiaoqian, S. Qiang, L. Jianguo, and L. Wenhua, "Capacitor Voltage Balancing Control based on CPS-PWM of Modular Multilevel Converter," in Energy Conversion Congress and Exposition (ECCE), 2011, pp. 4029-4034.

[4] D. Siemaszko, "Fast sorting method for balancing capacitor voltages in modular multilevel converters," IEEE Trans. Power Electron., vol. 30, no. 1, pp. 463-470, 2015.

[5] W. Yang, Q. Song, and W. Liu, "Decoupled Control of Modular Multilevel Converter Based on Intermediate," IEEE Trans. Ind. Electron., vol. 63, no. 8, pp. 4695-4706, 2016.

[6] G. P. Adam, K. H. Ahmed, S. J. Finney, and B. W. Williams, "AC fault ride-through capability of a VSC-HVDC transmission systems," in 2010 IEEE Energy Convers. Congr. Expo. ECCE 2010 - Proc., pp. 3739-3745, 2010.

[7] F. Sasongko, K. Sekiguchi, K. Oguma, M. Hagiwara, and H. Akagi, "Theory and Experiment on an Optimal Carrier Frequency of a Modular Multilevel Cascade Converter with Phase-Shifted PWM," IEEE Trans. Power Electron., vol. 31, no. 5, pp. 3456-3471, 2016.

[8] S. Fan, K. Zhang, J. Xiong, and Y. Xue, “An Improved Control System for Modular Multilevel Converters with New Modulation Strategy and Voltage Balancing Control," IEEE Trans. Power Electron., vol. PP, no. 99, pp. 358-371, 2014.

[9] N. R. Mehrabadi, R. Burgos, D. Boroyevich, and C. Roy, "Modeling and Design of the Modular Multilevel Converter with Parametric and Model-Form Uncertainty Quantification," in 2017 IEEE Energy Conversion Congress and Exposition (ECCE), 2017, pp. 1513-1520.

[10] A. Dekka, B. Wu, R. Lizana, and N. R. Zargari, "A comparison of voltage balancing versus energy balancing approach for modular multilevel converters," in IECON Proceedings (Industrial Electronics Conference), 2016, pp. 3117-3122.

[11] R. Zeng, L. Xu, L. Yao, and S. J. Finney, "Analysis and Control of Modular Multilevel Converters under Unbalanced Conditions," IEEE Trans. Ind. Electron., vol. 63, no. 1, pp. 71-81, 2016

[12] F. Zhang, C. Zhao, and J. Xu, "New control strategy of decoupling the AC/DC voltage offset for modular multilevel converter," IET Gener. Transm. Distrib. Spec., vol. 10, no. 6, pp. 1382-1392, 2016.

[13] R. Picas, S. Ceballos, J. Pou, J. Zaragoza, G. Konstantinou, and V. G. Agelidis, "Closed-loop discontinuous modulation technique for capacitor voltage ripples and switching losses reduction in modular multilevel converters," IEEE Trans. Power Electron., vol. 30, no. 9, pp. 4714-4725, 2015.

[14] S. Wang, G. P. Adam, A. M. Massoud, D. Holliday, and B. W. Williams, "Analysis and Assessment of Modular Multilevel Converter Internal Control Schemes," IEEE J. Emerg. Sel. Top. Power Electron., 2019.

[15] M. Vasiladiotis, N. Cherix, and A. Rufer, "Accurate Capacitor Voltage Ripple Estimation and Current Control Considerations for GridConnected Modular Multilevel Converters," IEEE Trans. Power Electron., vol. 29, no. 9, pp. 4568-4579, 2014. 